\title{
Trombose muito tardia de stent coronário não farmacológico: identificando má aposição e expansão por ultrassonografia intravascular
}

\author{
Very late stent thrombosis with bare-metal stent: identifying severe stent malapposition \\ and underexpansion by intravascular ultrasound
}

\author{
Adriano Caixeta ${ }^{1}$, Vanessa Cristina Salomon Palma Braga ${ }^{1}$, Gary S Mintz ${ }^{2}$
}

\section{RESUMO}

Paciente de 60 anos de idade, com histórico de implante de dois stents coronários não farmacológicos durante quadro de infarto do miocárdio com supra do segmento-ST. Dois anos após, foi admitido no pronto-socorro com quadro clínico de angina instável de início recente. 0 paciente fazia uso regular de inibidor da enzima de conversão da angiotensina, betabloqueador e aspirina. A aspirina foi suspensa por 10 dias antes da atual hospitalização para a retirada de um tumor de rim. 0 paciente foi submetido a angiografia coronária, que revelou artéria coronária direita com defeito de enchimento intraluminal sugestivo de trombo intra-stent. A ultrassonografia intravascular demonstrou má aposição e expansão dos stents, além da presença de trombo. Neste caso, o mecanismo de trombose muito tardia de stent coronário não farmacológico deveu-se possivelmente a uma combinação de fatores mecânicos (implante de stents subdimensionados durante o procedimento índice) e farmacológico (descontinuação da aspirina).

Descritores: Stents; Infarto do miocárdio; Trombose/ultrassonografia; Relatos de casos

\begin{abstract}
A 60-year-old man with a history of implantation of two bare-metal stents 2 years prior presented to the emergency department with new-onset chest pain. He has been regularly taking angiotensinconverting enzyme inhibitors, beta blockers and aspirin. Aspirin was suspended for 10 days prior to the current hospitalization in order to perform surgery to remove a kidney tumor. He underwent coronary angiography, which revealed a right coronary artery with a distal intraluminal defect within the stents, suggesting thrombus. Intravascular ultrasound demonstrated a severe malapposition and
\end{abstract}

underexpansion throughout the entire length of the stents containing thrombus. In this case, the mechanisms of very late stent thrombosis after bare-metal stent most likely were a combination of mechanical factors (severe stent undersizing during the index procedure) and pharmacological factors (aspirin discontinuation).

Keywords: Stents; Myocardial infarction; Thrombosis/ultrasonography; Case reports

\section{INTRODUÇÃo}

A intervenção coronária percutânea primária com implante de stent tem emergido como o tratamento de escolha para muitos pacientes com infarto agudo do miocárdio com supradesnivelamento do segmento ST (IAM com supra). Todavia, dados recentes sugerem que o risco de trombose de stent em pacientes com IAM com supra é relativamente aumentado quando comparado àqueles pacientes com IAM sem supra ${ }^{(1)}$ e ocorre com frequência similar com stents convencionais (SC) e stents farmacológicos $(\mathrm{SF})^{(2)}$. A endotelização e a cicatrização no local do implante do stent em pacientes com IAM com supra pode ser consideravelmente retardada ${ }^{(3)}$. Além disso, apesar de não haver diferença na frequência de trombose com os tipos diferentes de stents, as taxas relativamente alta de trombose muito tardia de stent convencionais recentemente registrada ${ }^{(2)}$ é notória e rejeita o conceito primário $^{(4)}$ de que a trombose muito tardia de stent convencional é um fenômeno raro. Além disso, são raros os dados e as imagens documentando o mecanismo de trombose

\footnotetext{
1 Hospital Israelita Albert Einstein, São Paulo, SP, Brasil.
}

${ }^{2}$ Cardiovascular Research Foundation, New York, USA.

Autor correspondente: Adriano Caixeta - Hospital Israelita Albert Einstein, Cardiologia Intervencionista, Avenida Albert Einstein, 627/701 - Morumbi - CEP: 05652-900 - São Paulo, SP, Brasil Tel.: (11) 2151-0453 - E-mail: adriano.caixeta@einstein.br 
muito tardia de stent em pacientes com IAM com supra. O objetivo deste caso foi relatar os mecanismos potenciais de trombose muito tardia de stent convencional por meio de ultrassonografia intravascular (USIV).
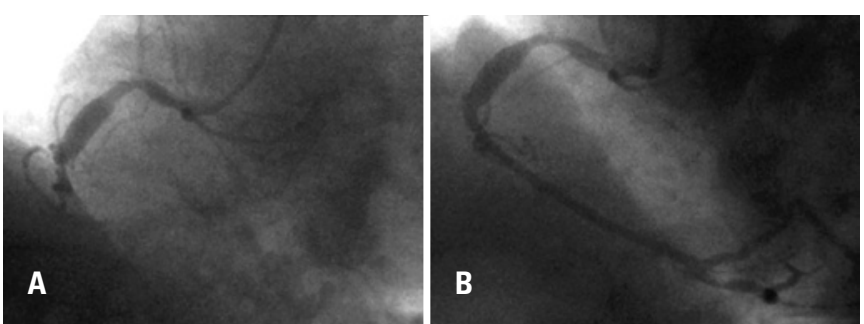

Figura 1. Angiografia coronária mostrando artéria coronária direita antes (A) e depois (B) da angioplastia coronária primária com implante de stents 2 anos antes da admissão hospitalar atual do paciente

\section{RELATO DE CASO}

Paciente de 60 anos, do gênero masculino, com histórico de implante de dois SC $(2,25 \times 28 \mathrm{~mm}$ e $2,5 \times 28 \mathrm{~mm})$ devido a IAM com supra inferior (Figura 1) 2 anos antes de ser admitido no departamento de emergência com angina instável de início recente. O eletrocardiograma revelou alterações na parede inferior e os marcadores creatina quinase-MB e troponina I estavam elevados em $3,03 \mathrm{ng} / \mathrm{mL}$ e $11,10 \mathrm{ng} / \mathrm{mL}$, respectivamente. O paciente fazia uso regular, em domicílio, de inibidores da enzima de conversão da angiotensina, betabloqueadores e aspirina, sendo que o último foi suspenso por 10 dias antes da hospitalização atual para realização de procedimento cirúrgico de retirada de tumor renal. $\mathrm{O}$ paciente foi submetido a angiografia coronária que revelou artéria coronária direita com ectasia difusa, contendo duas
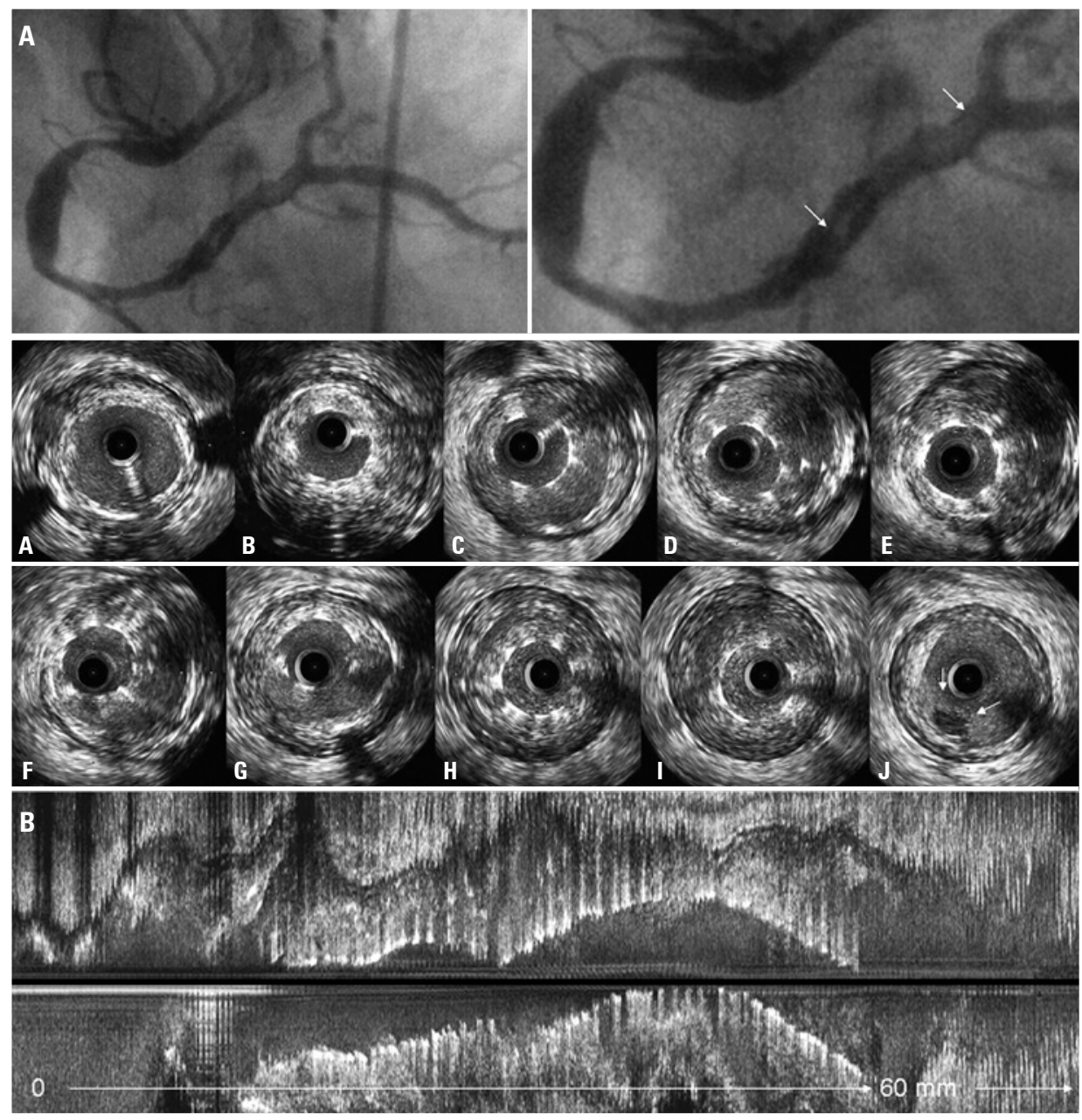

Figura 2. Ultrassonografia intravascular diagnóstica para avaliar o defeito no enchimento angiográfico na artéria coronária direita (A, setas brancas na angiografia). As imagens da ultrassonografia intravascular são mostradas da porção proximal (A) a distal (J) na figura B. Nota-se má aposição e expansão das hastes dos stents em toda a sua extensão. Observa-se espaço entre a haste do stent e a íntima; além de trombo por trás das hastes do stent no corte axial (B até I) e longitudinal (painel inferior). No local de máxima má aposição do stent (I), a área do stent $\left(4,99 \mathrm{~mm}^{2}\right)$ é menor do que a do lúmen (15,22mm²) e do que a lâmina elástica externa $\left(26,64 \mathrm{~mm}^{2}\right)$. 0 segmento completo de $+20 \mathrm{~mm}$ do stent foi preenchido por trombo; nota-se trombo adicional no lado abluminal do stent enchendo parcialmente a área de má aposição causando o defeito de enchimento linear ao angiograma. Uma pequena massa intraluminal na ultrassonografia intravascular (J, setas menores) representa a cauda do trombo com a porção mais robusta localizada proximalmente 
lesões (proximal e médio da artéria coronária direita) e defeito de enchimento intraluminal distal nos stents (Figura 2A, setas brancas), sugerindo trombo. A USIV (iLab, Boston Scientific, USA) das lesões mostrou grande vaso distal, com má aposição e expansão dos stents com presença de trombo (Figura 2B).

\section{DISCUSSÃO}

Apesar de se tratar de evento clínico de frequência relativamente baixa, a trombose de stent é associada com um alto risco de infarto do miocárdio e morte ${ }^{(5)}$. O risco de trombose de stent na população com IAM com supra é três vezes maior em relação a pacientes que não sofreram IAM com supra ${ }^{(1)}$ e ocorre com frequência similar em SC e SF(2). A mais alta taxa de trombose de stent em pacientes com IAM com supra pode estar relacionada à composição da placa subjacente responsável por esta condição. A penetração das hastes do stent em núcleo necrótico subjacente pode aumentar o processo inflamatório, a ativação plaquetária, o depósito de fibrina e inibição de crescimento neointimal, o que resulta em stent com hastes não recobertas ${ }^{(3)}$. Além disso, o remodelamento arterial positivo e aposição incompleta tardia adquirida do stent é maior em pacientes com síndromes coronárias agudas, especialmente com SF. Por fim, a vasoconstrição da artéria relacionada ao infarto, o fluxo epicárdico reduzido e a presença de trombo pode levar ao subdimensionamento do stent, como provavelmente ocorreu neste caso na ocasião do tratamento do IAM com supra onde um vaso de $4,5 \mathrm{~mm}$ foi tratado com stent de $2,5 \mathrm{~mm}$. Com passar do tempo, quando o fluxo foi restabelecido, o trombo dissolveu-se, o vaso aumentou de tamanho, desenvolvendo-se má aposição e expansão do stent. Neste relato, os mecanismos envolvidos na trombose muito tardia de stent convencional provavelmente resultaram da combinação de fatores mecânicos (subdimensionamento grave do stent durante o procedimento índice, com ou sem remodelamento positivo superposto, e dissolução do trombo relacionado ao infarto durante os 2 anos de seguimento) e farmacológicos (a descontinuação da aspirina).

\section{CONCLUSÃO}

Este relato de caso descreve os mecanismos de trombose muito tardia de stent não farmacológico 2 anos após o seu implante. A ultrassonografia intravascular tem um papel importante na identificação dos fatores mecânicos relacionados à trombose de stent, além de ser útil como guia na otimização da terapia mais apropriada.

\section{REFERÊNCIAS}

1. Lagerqvist B, Carlsson J, Fröbert O, Lindbäck J, Scherstén F, Stenestrand U, James SK; Swedish Coronary Angiography and Angioplasty Registry Study Group. Stent thrombosis in Sweden: a report from the Swedish Coronary Angiography and Angioplasty Registry. Circ Cardiovasc Interv. 2009;2(5): 401-8.

2. Dangas GD, Caixeta A, Mehran R, Parise H, Lansky AJ, Cristea E, Brodie BR, Witzenbichler B, Guagliumi G, Peruga JZ, Dudek D, Möeckel M, Stone GW; Harmonizing Outcomes With Revascularization and Stents in Acute Myocardial Infarction (HORIZONS-AMI) Trial Investigators. Frequency and predictors of stent thrombosis after percutaneous coronary intervention in acute myocardial infarction. Circulation. 2011;123(16): 1745-56.

3. Nakazawa G, Finn AV, Joner M, Ladich E, Kutys R, Mont EK, et al. Delayed arterial healing and increased late stent thrombosis at culprit sites after drugeluting stent placement for acute myocardial infarction patients: an autopsy study. Circulation. 2008;118(11):1138-45.

4. Mauri L, Hsieh WH, Massaro JM, Ho KK, D’Agostino R, Cutlip DE. Stent thrombosis in randomized clinical trials of drug-eluting stents. N Engl J Med. 2007;356(10):1020-9.

5. lakovou I, Schmidt T, Bonizzoni E, Ge L, Sangiorgi GM, Stankovic G, et al. Incidence, predictors, and outcome of thrombosis after successful implantation of drug-eluting stents. JAMA. 2005;293(17):2126-30. 